\title{
Finite-size, chemical-potential and magnetic effects on the phase transition in a four-fermion interacting model
}

\author{
E. B. S. Corrêa ${ }^{1,3, a}$, C. A. Linhares ${ }^{2, b}$, A. P. C. Malbouisson ${ }^{3, c}$, J. M. C. Malbouisson ${ }^{4, d}$, A. E. Santana ${ }^{5, \mathrm{e}}$ \\ ${ }^{1}$ Instituto de Ciências Exatas, Universidade Federal do Sul e Sudeste do Pará, Marabá 68505-080, Brazil \\ ${ }^{2}$ Instituto de Física, Universidade do Estado do Rio de Janeiro, Rio de Janeiro 20559-900, Brazil \\ ${ }^{3}$ Centro Brasileiro de Pesquisas Físicas-CBPF/MCTI, Rio de Janeiro 22290-180, Brazil \\ ${ }^{4}$ Instituto de Física, Universidade Federal da Bahia, 40210-340 Salvador, Brazil \\ ${ }^{5}$ Instituto de Física, Universidade de Brasília, 70910-900 Brasília, DF, Brazil
}

Received: 1 December 2016 / Accepted: 10 April 2017 / Published online: 25 April 2017

(C) The Author(s) 2017. This article is an open access publication

\begin{abstract}
We study effects coming from finite size, chemical potential and from a magnetic background on a massive version of a four-fermion interacting model. This is performed in four dimensions as an application of recent developments for dealing with field theories defined on toroidal spaces. We study effects of the magnetic field and chemical potential on the size-dependent phase structure of the model, in particular, how the applied magnetic field affects the size-dependent critical temperature. A connection with some aspects of the hadronic phase transition is established.
\end{abstract}

\section{Introduction}

The need to construct effective models sharing properties with realistic theories comes from the very involved mathematical structure of these last ones. For instance, this occurs in studies of quantum chromodynamics, which practically prevents us from finding analytical results taking into account both confinement and asymptotic freedom. Rigorous calculations in this case, both at zero and finite temperature, have been worked out $[1,2]$, but mainly treating the asymptotically-free domain at high energies or high temperatures, where perturbation theory is applicable.

That is why simplified, phenomenological models have been implemented along the years. Among them, one of the simpler and most successful is the Gross-Neveu (GN)

\footnotetext{
a e-mail: ecorrea@unifesspa.edu.br

be-mail: linharescesar@gmail.com

c e-mail: adolfo@cbpf.br

de-mail: jmalboui@ufba.br

e e-mail: asantana@unb.br
}

model [3], considered as an effective theory for many situations in condensed-matter and in hadronic physics [4-18]. It has proved to be an enlightening approach in describing properties of superconductors and graphene in condensed matter physics [5-8] and also phenomena in hadronic matter, such as spatial and thermal asymptotic freedom and the spatial and thermal confinement/deconfinement phase transition [13-18]. The GN model provides the simplest effective theory which may be considered as describing interactions between fermions, as a direct four-body coupling, where gauge fields and degrees of freedom are integrated out. As such, one needs not to worry about small values of the coupling constant to perform perturbative summations, nor with renormalizability. That is why we consider just a one-loop correction to the mass. In this spirit, perturbative renormalizability is not an absolute requirement for an effective theory to be a physically meaningful model; see for instance [1922]. In this context, the massive Gross-Neveu model can be studied in an arbitrary dimension $D$, although it is not renormalizable for $D>2$. Models of this type, including chemical-potential effects in an arbitrary dimension $D$, are already present in the literature [23].

A recent work dealing with a large- $N$ four-fermion interacting model in arbitrary dimension $D$ is in Ref. [16]; this has been done by an analysis of the four-point function at criticality, taking inspiration from linear response theory and the BCS theory of superconductivity [24]. We here approach the GN model in a non-standard way. As in [25], we deal with a one-component version of a four-fermion interacting model. Most importantly, instead of working directly with the usual Dirac spinor degrees of freedom, a grand-canonical thermal average $\left\langle\psi^{\dagger}(x) \psi(x)\right\rangle$ of the product of fermion fields is performed, and an Ansatz is made by considering, to our purposes, a free-energy density written in terms of such aver- 
ages. This approach has been previously used for instance in Refs. [25,26]

In the present note, we determine a phase-transition temperature for the model and investigate how this temperature is affected by the presence of an external magnetic field, the chemical potential and the finite size of the system. The study performed here may be viewed as a fermionic counterpart, including magnetic effects, of corresponding investigations on the phase transition for scalar theories [26,27], using general methods of quantum field theories defined on spaces with toroidal topologies [28-30].

The general framework is the field-theoretical model defined on a Euclidean $D$-dimensional space, under the influence of an external magnetic field $B$. We impose antiperiodic boundary conditions on the time coordinate of length $\beta=T^{-1}$, the inverse temperature, and also on a spatial coordinate of length $L$; as is argued for instance in [28], this is equivalent to consider the system limited by two parallel planes orthogonal to the compactified spatial coordinate a distance $L$ apart from one another (the size of the system). Then we study concurrently effects of the chemical potential and of the applied external magnetic field on the size-dependent phase structure for the second-order transition occurring in the system. We will consider an applied magnetic field along the compactified spatial axis. In this case, an effect of the external field is to break the symmetry over two of the space dimensions orthogonal to the magnetic field, leaving a two-dimensional translationally invariant space. This implies that the space dimensionality should be at least $D \geq 4$. We restrict ourselves to the $D=4$ case. Notice that our system is strictly $(3+1)$-dimensional, with three spatial dimensions, one of these corresponding to the thickness of the system, so that we can establish, from our formulas, the tendency to the bulk critical temperature as the thickness grows. But we cannot consider this as an infinitely short length, the system we study has a third dimension which plays an important role, and should not be neglected. From our point of view, the system cannot and should not be reduced to a $(2+1)$-dimensional system.

One of the conclusions is that no transition exists below a minimal size of the system. This size depends on the chosen model, i.e., on the value of the coupling constant we take and on the intensity of the applied field. In any case, this is understandable from a physical point of view, since long-range correlations cannot persist at very small distances. We consider heuristically our model as describing a system of fermion-antifermion pairs, a distance $L$ apart from one another and the transition temperature we find is interpreted as the temperature at which the pairs dissociate. In this case, dissociation of the system is favoured by stronger applied fields and larger values of the coupling constant. An interesting aspect of the results is that the sizes involved and the critical temperatures obtained, are compatible with charac- teristic quantities in particle physics, e.g., the size of a meson and the deconfining hadronic temperature.

\section{Magnetic effects on the model}

We consider the massive Gross-Neveu model in a $D$ dimensional Euclidean manifold, $\mathbb{R}^{D}$, at zero chemical potential, described by the Hamiltonian

$H=\int \mathrm{d}^{D} x\left(\psi^{\dagger}(x)\left(i \gamma_{\mu} D_{\mu}-m_{0}\right) \psi(x)+\frac{\lambda_{0}}{2}\left(\psi^{\dagger}(x) \psi(x)\right)^{2}\right)$,

where $m_{0}$ and $\lambda_{0}$ are respectively the physical mass and coupling constant at zero temperature and zero chemical potential in the absence of boundaries and of an applied magnetic field. In the above equation, $D_{\mu}=\partial_{\mu}-i e A_{\mu}$ is the covariant derivative, and the gauge $A_{\mu}=\left(0,0, B x_{1}, \ldots, 0\right)$ is adopted. A constant and uniform magnetic field $B$ is applied along the $x_{3}$-direction ( $z$ axis). The $\gamma$-matrices are elements of the Clifford algebra and we use natural units, $\hbar=c=k_{B}=1$. This Hamiltonian is based in conventions for Euclidean field theories as presented in Ref. [31]. Dimensional analysis of Eq. (1) shows that $\lambda_{0}$ has the dimension of (mass) ${ }^{2-D}$.

Let us introduce in the model finite-temperature $\beta^{-1}$ and chemical-potential $\mu$ corrections to the mass, considering one spatial dimension compactified with a compactification length $L$. In the spirit of effective theories, we introduce one-loop thermal and boundary corrections to $m_{0}$ and define the temperature-, chemical-potential-, magnetic-field-, and boundary-dependent mass, $m(D, \beta, L, \mu, \omega)$, by

$m(D, \beta, L, \mu, \omega)=m_{0}+\Sigma(D, \beta, L, \mu, \omega)$,

where we have defined $\omega=e B$, which is the so-called cyclotron frequency.

As we have mentioned in the Introduction, we now make the following Ansatz: the fermionic fields in the Hamiltonian of Eq. (1) are submitted to a thermal average in the grand-canonical ensemble, denoted by $\langle\cdot\rangle$. We then define $\varphi(x)=\sqrt{\left\langle\psi^{\dagger}(x) \psi(x)\right\rangle}$ and make the approximation that the average of the square of the product of two fields is the square of the respective average of the two fields, namely, $\left\langle\left(\psi^{\dagger}(x) \psi(x)\right)^{2}\right\rangle \approx\left\langle\psi^{\dagger}(x) \psi(x)\right\rangle^{2}=\varphi^{4}(x)$. As a consequence, our model assumes the form of an effective freeenergy density of the Ginzburg-Landau type,

$f(\varphi)=-m(D, \beta, L, \mu, \omega) \varphi^{2}+\lambda_{0} \varphi^{4}$.

In an analogous way as in Refs. [25,26], we consider that all dependence on the physical parameters (temperature, chemical potential, finite size, external applied field) are concentrated in the coefficient of $\varphi^{2}$ in the free energy. The new field $\varphi(x)$ is interpreted as the order parameter for the transition. 
Notice that the thermally corrected mass in Eqs. (2) and (3) incorporates the chemical potential automatically, by the use of the appropriate Matsubara rule for introducing temperature in the grand-canonical ensemble.

The minus sign for the mass in Eq. (3) implies that, in the disordered phase, we have $m(D, \beta, L, \mu, \omega)<0$ and, for the ordered phase, $m(D, \beta, L, \mu, \omega)>0$. A second-order phase transition occurs for the set of points in the $(\beta, L, \mu, \omega)$ -space where $m(D, \beta, L, \mu, \omega)=0$, characterizing a spontaneous symmetry breaking.

The influence of a constant external magnetic field on the fermionic propagator can be completely determined through the eigenvalue method devised by Ritus a few decades ago [32,33], on which a renewed interest appeared in more recent years $[34,35]$. It uses some $\omega$-dependent eigenfunctions $E_{p}(x)$ of the covariant Dirac operator. They provide a kind of Fourier transform to diagonalize a momentum space which has lost translation invariance due to the presence of the magnetic field along a definite direction. The elegance of the Ritus method lies in that the magnetic-fielddependent propagator results in the same form of the free one. Its Euclidean version reads

$S(\bar{p})=\sum_{\ell=0}^{\infty} \frac{\omega}{2 \pi} \frac{\left(\not p-m_{0}\right)}{\bar{p}^{2}+m_{0}^{2}}$

where the momentum 4-vector is replaced by the $\omega$ dependent vector $\bar{p}$ which is

$\bar{p}_{\mu}=\left(p_{0}, \bar{p}_{1}, \bar{p}_{2}, p_{3}\right)$,

with

$\bar{p}^{2}=p_{0}^{2}+p_{3}^{2}+\bar{p}_{1}^{2}+\bar{p}_{2}^{2} ; \quad \bar{p}_{1}^{2}+\bar{p}_{2}^{2}=\omega(2 \ell+1-\sigma)$.

Here $\sigma= \pm 1$ is the spin variable. Also, one sees that the integer parameter $\ell$ identifies the Landau levels. If one is interested just in strong magnetic fields, only the lower level $(\ell=0)$ may be taken into account. In the present paper, we shall keep the contribution of all levels in our calculations.

\section{The corrected mass with an applied magnetic field}

In the presence of an external magnetic field, before introducing temperature and spatial compactification, the corrected mass is written as

$m(\omega)=m_{0}[1+\Sigma(\omega)]$,

where the self-energy $\Sigma$, for $D=4$, at one-loop order is given by

$\Sigma(\omega)=-\frac{\lambda_{0}}{m_{0}} \sum_{\sigma= \pm 1} \int \frac{\mathrm{d}^{2} \bar{p}}{(2 \pi)^{2}} \operatorname{tr}\left[\sum_{\ell=0}^{\infty} \frac{\omega\left(\not p-m_{0}\right)}{2 \pi\left(\bar{p}^{2}+m_{0}^{2}\right)}\right]$,

with the integration being over the variables $p_{0}$ and $p_{3}$.

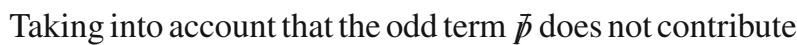
to the integral, Eq. (8) can be rewritten, in a convenient form for analytic regularization, as

$$
\begin{aligned}
\Sigma(\omega)= & \frac{2 \lambda_{0} \omega}{\pi} \sum_{\sigma= \pm 1} \sum_{\ell=0}^{\infty} \int \frac{\mathrm{d}^{2} \bar{p}}{(2 \pi)^{2}} \\
& \times\left.\frac{1}{\left[\bar{p}_{0}^{2}+\bar{p}_{3}^{2}+\omega(2 \ell+1-\sigma)+m_{0}^{2}\right]^{\nu}}\right|_{\nu=1} .
\end{aligned}
$$

Finite-temperature and density (chemical potential) corrections to the self-energy, together with the compactification of one of the spatial dimensions, are taken into account by using the appropriate generalized Matsubara formalism, i.e., the Feynman rules are modified accordingly to [28-30,36]

$\int \frac{\mathrm{d} \bar{p}_{0}}{2 \pi} \rightarrow \frac{1}{\beta} \sum_{n_{1}=-\infty}^{+\infty}, \quad \bar{p}_{0} \rightarrow \omega_{n_{1}}-i \mu ;$

$\int \frac{\mathrm{d} \bar{p}_{3}}{2 \pi} \rightarrow \frac{1}{L} \sum_{n_{2}=-\infty}^{+\infty}, \quad \bar{p}_{3} \rightarrow \omega_{n_{2}}$

where $\omega_{n_{1}}=\left(2 n_{1}+1\right) \pi / \beta$ and $\omega_{n_{2}}=\left(2 n_{2}+1\right) \pi / L$ are generalized Matsubara frequencies. Using this recipe, the self-energy becomes

$$
\begin{aligned}
\Sigma(t, \xi, \gamma, \delta)= & \frac{\lambda \delta t \xi}{2 \pi^{3}} \sum_{\sigma= \pm 1} \sum_{\ell=0}^{\infty} \sum_{n_{1}, n_{2}=-\infty}^{+\infty} \\
& \times\left.\frac{1}{\left[a_{1}\left(n_{1}-b_{1}\right)^{2}+a_{2}\left(n_{2}-b_{2}\right)^{2}+c_{\ell}^{2}\right]^{\nu}}\right|_{\nu=1},
\end{aligned}
$$

where we have defined the dimensionless coupling constant $\lambda$, the reduced temperature $t$, the reduced chemical potential $\gamma$, the reduced inverse length of the system $\xi$, and the reduced magnetic field $\delta$, in such a way that we have the set of dimensionless parameters defined by

$\lambda=\lambda_{0} m_{0}^{2}, t=\frac{T}{m_{0}}, \xi=\frac{L^{-1}}{m_{0}}, \delta=\frac{\omega}{m_{0}^{2}}, \gamma=\frac{\mu}{m_{0}}$,

and

$c_{\ell}^{2}=c_{\ell}^{2}(\delta, \sigma)=\frac{\delta(2 \ell+1-\sigma)+1}{4 \pi^{2}}, \quad a_{1}=\left(m_{0} \beta\right)^{-2}=t^{2} ;$

$a_{2}=\left(m_{0} L\right)^{-2}=\xi^{2}, \quad b_{1}=i \beta \mu / 2 \pi-1 / 2=i \gamma / 2 \pi t-1 / 2 ;$

$b_{2}=-1 / 2$.

The double sum over $n_{1}$ and $n_{2}$ in Eq. (11) is recognized as one of the two-variable Epstein-Hurwitz zeta function, defined by [37]

$$
\begin{aligned}
Z_{2}^{c_{\ell}^{2}}\left(v ; a_{1}, a_{2} ; b_{1}, b_{2}\right)= & \sum_{n_{1}, n_{2}=-\infty}^{\infty} \\
& \times\left[a_{1}\left(n_{1}-b_{1}\right)^{2}+a_{2}\left(n_{2}-b_{2}\right)^{2}+c_{\ell}^{2}\right]^{-v} .
\end{aligned}
$$


This Epstein-Hurwitz zeta function has an analytical continuation, valid in the whole complex $v$-plane [37], and can be written as [26,27]

$$
\begin{aligned}
Z_{2}^{c_{\ell}^{2}}\left(v ;\left\{a_{j}\right\} ;\left\{b_{j}\right\}\right)= & \frac{\pi\left[c_{\ell}(\delta, \sigma)\right]^{2-2 v}}{t \xi} \frac{\Gamma(v-1)}{\Gamma(v)} \\
& +\frac{4 \pi^{v}\left[c_{\ell}(\delta, \sigma)\right]^{1-v}}{t \xi \Gamma(v)} \mathscr{R}_{c_{\ell}(\delta, \sigma)}(\nu ; t, \xi, \gamma),
\end{aligned}
$$

where

$$
\begin{aligned}
\mathscr{R}_{c_{\ell}(\delta, \sigma)}(v ; t, \xi, \gamma)= & \sum_{n_{1}=1}^{\infty}(-1)^{n_{1}} \cosh \left(\frac{n_{1} \gamma}{t}\right)\left(\frac{n_{1}}{t}\right)^{\nu-1} \\
& \times K_{1-v}\left(\frac{2 \pi c_{\ell}(\delta, \sigma) n_{1}}{t}\right) \\
& +\sum_{n_{2}=1}^{\infty}(-1)^{n_{2}}\left(\frac{n_{2}}{\xi}\right)^{v-1} \\
& \times K_{1-v}\left(\frac{2 \pi c_{\ell}(\delta, \sigma) n_{2}}{\xi}\right) \\
& +2 \sum_{n_{1}, n_{2}=1}^{\infty}(-1)^{n_{1}+n_{2}} \cosh \left(\frac{n_{1} \gamma}{t}\right) \\
& \times\left(\sqrt{\frac{n_{1}^{2}}{t^{2}}+\frac{n_{2}^{2}}{\xi^{2}}}\right)^{v-1} \\
& \times K_{1-v}\left(2 \pi c_{\ell}(\delta, \sigma) \sqrt{\frac{n_{1}^{2}}{t^{2}}+\frac{n_{2}^{2}}{\xi^{2}}}\right) .
\end{aligned}
$$

The first term in the representation of the zeta function presented in Eq. (14) is divergent for $v \rightarrow 1$, while the second term is regular in this limit. To get a finite self-energy, we implement a minimal subtraction by discarding this singular term. Actually, we make an expansion around $v=1$, which leads to a pole term at $v=1$, plus a logarithmic term in $c_{\ell}(\delta, \sigma)$, and finite terms. Using the definition of $c_{\ell}(\delta, \sigma)$ and summing over the spin polarizations, and taking into account all Landau levels, it can be shown that this logarithmic contribution is divergent, no matter how small be the applied field. In this case, this contribution should be subtracted along with the pole term to get a finite mass. Such a procedure is equivalent to taking out the whole first term in Eq. (14).

Substituting Eq. (14) in Eq. (11), after subtraction of the divergent term, we get a finite correction to the mass,

$\Sigma_{R}(t, \xi, \gamma, \delta)=\frac{2 \lambda \delta}{\pi^{2}} \sum_{\sigma= \pm 1} \sum_{\ell=0}^{\infty} \mathscr{R}_{c_{\ell}(\delta, \sigma)}(1 ; t, \xi, \gamma)$,

where $\mathscr{R}_{c_{\ell}(\delta, \sigma)}(1 ; t, \xi, \gamma)$ is given by Eq. (15).
The corrected mass is obtained by taking Eq. (16) into Eq. (7). Performing the summation over the spin polarizations, we obtain

$$
\begin{aligned}
\frac{m(t, \xi, \gamma, \delta)}{m_{0}}=1 & +\frac{2 \lambda \delta}{\pi^{2}} \\
& \times\left[\mathscr{F}_{0}(t, \xi, \gamma, \delta)+2 \sum_{\ell=1}^{\infty} \mathscr{F}_{\ell}(t, \xi, \gamma, \delta)\right],
\end{aligned}
$$

where

$$
\begin{aligned}
\mathscr{F}_{\ell}(t, \xi, \gamma, \delta)= & \sum_{n_{1}=1}^{\infty}(-1)^{n_{1}} \cosh \left(\frac{n_{1} \gamma}{t}\right) K_{0}\left(\frac{\sqrt{2 \delta \ell+1} n_{1}}{t}\right) \\
& +\sum_{n_{2}=1}^{\infty}(-1)^{n_{2}} K_{0}\left(\frac{\sqrt{2 \delta \ell+1} n_{2}}{\xi}\right) \\
& +2 \sum_{n_{1}, n_{2}=1}^{\infty}(-1)^{n_{1}+n_{2}} \cosh \left(\frac{n_{1} \gamma}{t}\right) \\
& \times K_{0}\left(\sqrt{2 \delta \ell+1} \sqrt{\frac{n_{1}^{2}}{t^{2}}+\frac{n_{2}^{2}}{\xi^{2}}}\right)
\end{aligned}
$$

The expression in Eq. (18) has a well-defined meaning for values of the reduced chemical potential in the interval $0 \leq \gamma<1$. The sums in Eq. (18) also occur in formulas obtained in the study of the bosonic $\phi^{4}$ theory [26] under the same physical conditions (that is, presence of chemical potential and external magnetic field, and size limitation). As argued in Ref. [26], the large- $n_{1}$ limit allows us to employ the asymptotic form of the Bessel function, that is, $K_{0}(z)=\sqrt{\pi / 2 z} e^{-z}$, for large $z$. In the case of the first sum of $\mathscr{F}_{\ell}$, the argument of the function is $z=\frac{n_{1}}{t} \sqrt{2 \delta \ell+1}$, which, together with the definition of the hyperbolic cosine, leads to an expression proportional to $\exp \left[-\frac{n_{1}}{t}(\sqrt{2 \delta \ell+1}-\gamma)\right]+$ $\exp \left[-\frac{n_{1}}{t}(\sqrt{2 \delta \ell+1}+\gamma)\right]$. The last term, which gives a convergent sum over $n_{1}$, is of no consequence to the possible values of $\gamma \geq 0$; however, the first one implies convergence only when $0 \leq \gamma<\sqrt{2 \delta \ell+1}$. And, in fact, if one wishes to consider arbitrarily vanishing of the applied magnetic field, the restriction on the chemical potential becomes $0 \leq \gamma<1$. The same conclusion comes about for the last sum in Eq. (18) and also for $\mathscr{F}_{0}$.

Criticality is attained when the corrected mass, given by Eq. (17), vanishes. The solutions of $m(t, \xi, \gamma, \delta)=0$ provide the size-dependent critical temperatures as a function the applied magnetic field.

An entirely analogous (and simpler) calculation leads to the critical equation in the absence of an applied field but, in this case, an integral over two momentum variables (those whose symmetry over the corresponding coordinates is broken by the magnetic field) should be evaluated using dimensional regularization methods; one obtains [38] 


$$
\frac{m(t, \xi, \gamma)}{m_{0}}=1+\frac{2 \lambda}{\pi^{2}} \mathscr{R}_{c}(0 ; t, \xi, \gamma),
$$

in this case, $c=1 / 2 \pi$.

\section{Phase structure}

In Fig. 1, we plot a set of curves that give the reduced critical temperature, $t_{c}$, as a function of the reduced inverse size of the system $\xi$, for three values of the reduced chemical potential: $\gamma=0.0,0.35$, and 0.7 , corresponding to the full, dotdashed and dotted lines respectively; we take a fixed value of the reduced applied field, $\delta=8.0$ and the dimensionless coupling constant $\lambda=2.0$. For each value of $\gamma$ the broken phase is at the interior of the corresponding curve. In Fig. 2, we exhibit four curves for the reduced critical temperature, $t_{c}$, as a function of the reduced inverse size of the system, $\xi$, for vanishing chemical potential, $\gamma=0.0$, fixed $\lambda=1.0$ and some values of the magnetic field: $\delta=0.0,0.3,2.0$, and 7.0, full, dashed, dotdashed and dotted lines, respectively. Notice that the curve for vanishing applied field, full line in the Fig. 2, is obtained from Eq. (19), valid in the absence of an applied field.

From the figures we see that the qualitative behaviour of the size-dependent critical temperature has similarities with and without an applied field. In both cases there is a minimal size, $L_{0}$, corresponding to a reduced inverse size, $\xi_{0}$, for which the transition temperature vanishes. This minimal size

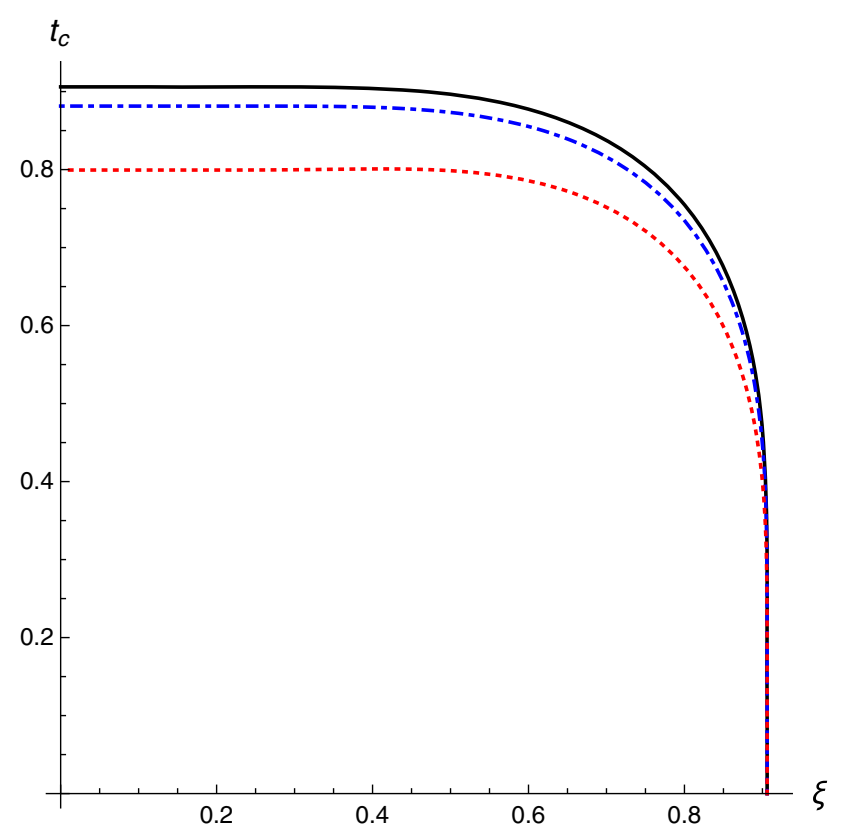

Fig. 1 Phase diagram $\left(\xi \times t_{c}\right)$ for fixed values of the magnetic field, $\delta=8.0$, and the coupling constant, $\lambda=2.0$, and three values of the chemical potential: $\gamma=0.0 ; 0.35$; and 0.7 , corresponding to the full, dotdashed and dotted lines respectively

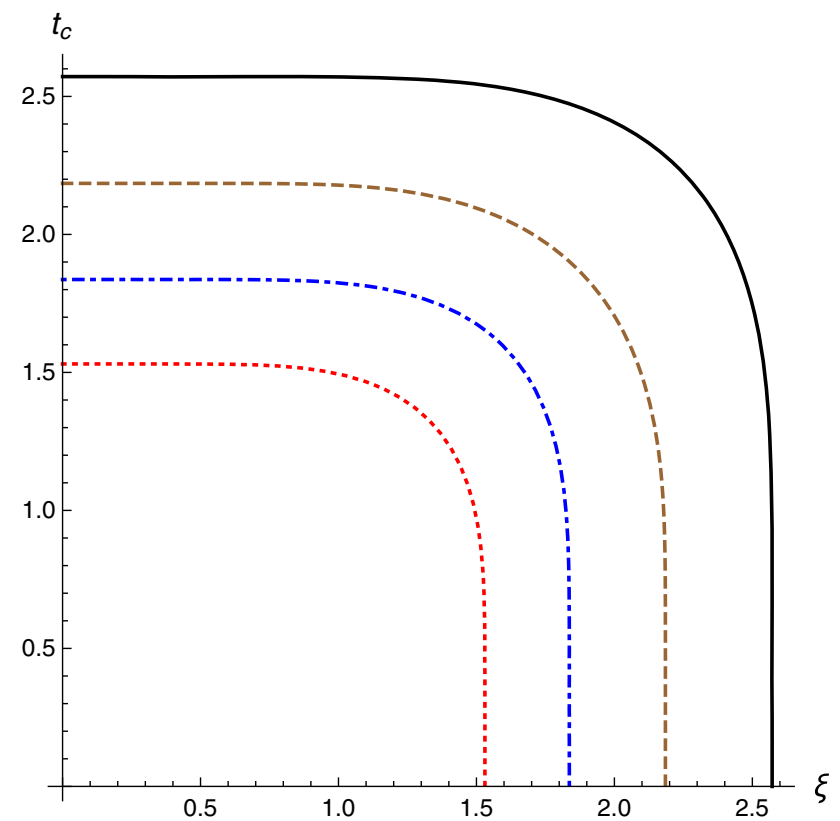

Fig. 2 Reduced critical temperature, $t_{c}$, as a function of the reduced inverse size of the system, $\xi$, for a vanishing chemical potential, $\gamma=$ 0.0 , fixed $\lambda=1.0$, and some values of the magnetic field: $\delta=0.0 ; 0.3$; 2.0; and 7.0, full, dashed, dotdashed and dotted lines, respectively

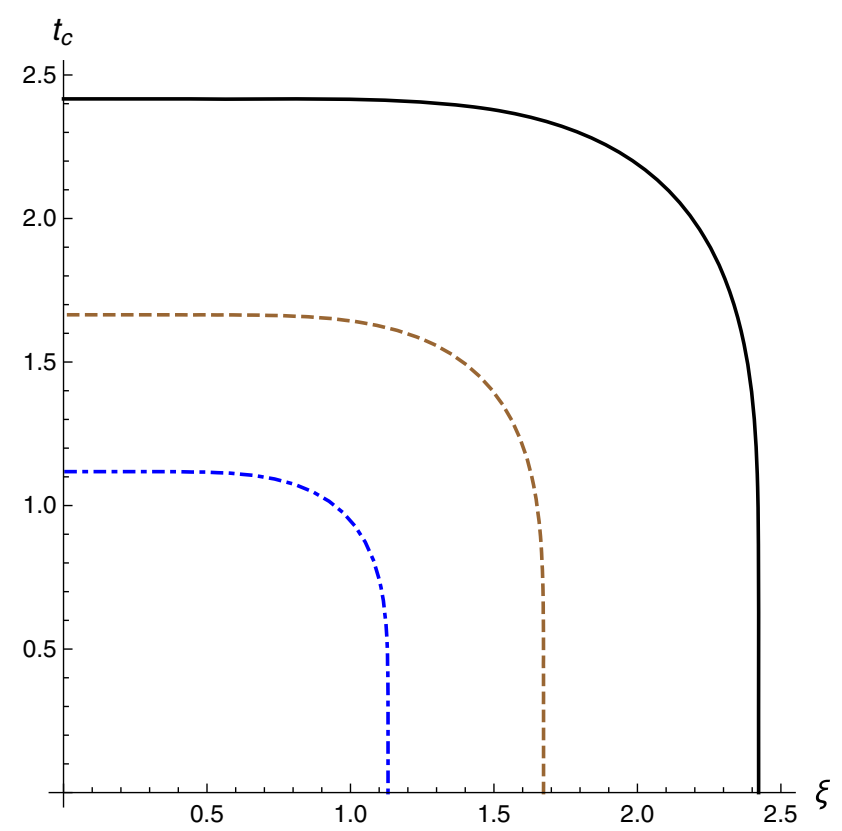

Fig. 3 Reduced transition temperature as a function of the reduced inverse size, for fixed $\gamma=0.3$ and $\delta=5.0$, and distinct values of the quartic self-coupling: $\lambda=0.5 ; 1.0$; and 2.0, full, dashed and dotdashed lines, respectively

appears to be independent of the chemical potential. Actually, this can be explicitly shown by taking the $t \rightarrow 0$ limit in the critical equation (17). A similar behaviour was found for a scalar field in Ref. [26]. We see that the behaviour of 


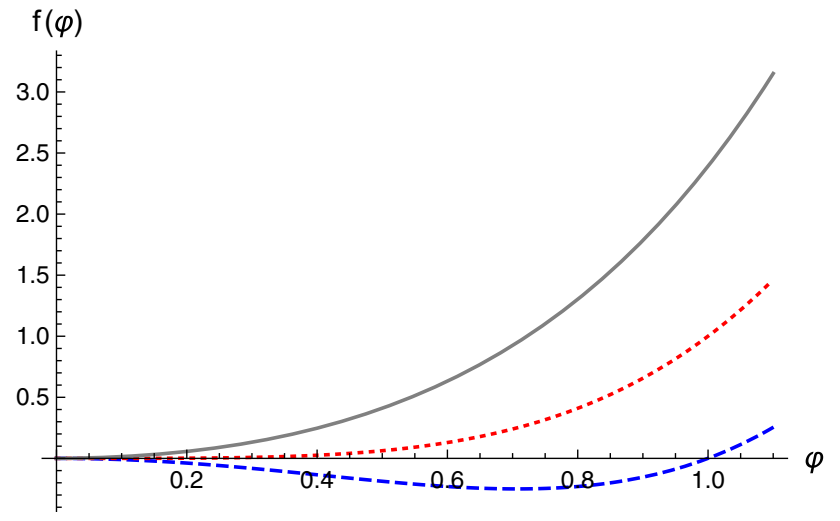

Fig. 4 Ginzburg-Landau free-energy density for fixed values: $\xi=$ $0.001, \gamma=0.35, \delta=7.0$ and $\lambda=2.0$. Disordered phase is represented by full curve, which has temperature equal 1.7. Critical temperature is got at $t_{c} \approx 0.95$ (dotted curve). Ordered phase is got below $t_{c}$. We have $t=0.01$ in dashed curve

the size-dependent critical temperature, illustrated in Fig. 1 shows that it is weakly dependent on the chemical potential and that this dependence is mainly concentrated for higher critical temperatures and larger sizes of the system. We notice also that the intensity of the quartic coupling has a significative influence on the behaviour of the system: As illustrated in Fig. 3, higher values of $\lambda$ leads to larger values of the minimal allowed size $L_{0}$ and lower values of the critical temperature. This reinforces the effect due to the magnetic field of lowering the critical temperature, as can be seen from Fig. 2. In Fig. 4, we show the free-energy behaviour for different temperatures; above $t_{c}$, the free-energy has a single absolute minimum at $\varphi=0$, while below $t_{c}$ the minimum occurs at a finite value, $\varphi_{0}(t)$, which goes continuously to zero as $t \rightarrow t_{c}$ from below, thus ensuring that the transition is a second order one.

\section{Comments}

As a central part of this work, we have analyzed joint effects due to an external applied field, chemical potential, and the finite size of the system, on a second-order phase transition. One of the conclusions is that no transition exists below a minimal size. This size depends on the chosen model, i.e., on the value of the coupling constant we take and on the intensity of the applied field. In any case, this is understandable from a physical point of view, since long-range correlations cannot persist at very small distances. The results in this note have been possible by employing the massive GN model as an effective theory. In previous works devoted to get insights into the behaviour of hadronic matter, the massless GN model, in its version with a large number of components, has been often employed. Using the one-component massive GN model and taking the fermion mass as a physical param- eter, we have been able, through analytical means, to study finite-size effects on the transition.

Let us adopt a heuristic approach, in which sense we think of the model as a simplified description (a "toy model") of a heated system of fermion-antifermion pairs of size $L$ in equilibrium at temperature $\beta^{-1}$. The transition temperature could be interpreted as the temperature at which the pairs dissociate.

Under this heuristic point of view, let us make some comments about our results. Let us first consider the system in the absence of an applied field. Using Eq. (12), we find a minimal size of the system of $L_{0}=1 / m_{0} \xi_{0}$. In this case, we get from Fig. 2 that at zero temperature and with $\gamma=0.0$ and $\lambda=1.0$, the reduced inverse size is roughly $\xi_{0} \approx 2.57$. Taking for $m_{0}$ the effective current quark mass of $\sim 68.3 \mathrm{MeV}$ [39] (which corresponds approximately to half of the mass of a pion), we obtain, using the conversion $\mathrm{MeV}^{-1} \approx 196.9 \mathrm{fm}$, $L_{0} \approx 1.12 \mathrm{fm}$; this is of the order of magnitude of the estimated size of a meson. On the other hand, we see from the full line in Fig. 2, that for reduced inverse sizes such that $\xi \leq 1.20$, the reduced temperature is almost constant and has a value of $t \approx 2.56$. This gives a transition temperature of $T_{c} \approx 175 \mathrm{MeV}$ for all sizes $L \geq 2.40 \mathrm{fm}$, much larger that the zero-temperature minimal size, which we think as being the size of a fermion-antifermion bound state. The transition temperature that we have is close to the estimated deconfining temperature for hadrons. We can think of this temperature as the temperature at which the mesons dissociate in the absence of an applied field. When an applied field is present, for instance, $\delta=2.0$, we find from Fig. $2, \xi_{0}(\delta=2.0) \approx 1.83$, corresponding to a minimal size of $L_{0}(\delta=2.0) \approx 1.58 \mathrm{fm}$. Comparing with the value $L_{0}(\delta=0.0) \approx 1.12 \mathrm{fm}$, we see that, even at zero temperature, the action of the magnetic field tends to dissociate the system. This effect is more important for stronger magnetic fields and higher temperatures. Also, dissociation of the system is favored for higher values of the coupling constant; as already mentioned above, larger values of the quartic coupling constant leads to larger values of the minimal allowed size $L_{0}$ and lower values of the critical temperature. A particularly interesting aspect of the results is that the sizes involved and the critical temperatures obtained, are compatible with characteristic quantities in particle physics, e.g., the size of a meson and the deconfining hadronic temperature.

Acknowledgements We thank financial support from Conselho Nacional de Desenvolvimento Científico e Tecnológico and Fundação de Amparo à Pesquisa do Estado do Rio de Janeiro, Brazilian agencies. EBSC also thanks Coordenação de Aperfeiçoamento de Pessoal de Nível Superior/PRODOUTORAL-UNIFESSPA program.

Open Access This article is distributed under the terms of the Creative Commons Attribution 4.0 International License (http://creativecomm ons.org/licenses/by/4.0/), which permits unrestricted use, distribution, and reproduction in any medium, provided you give appropriate credit 
to the original author(s) and the source, provide a link to the Creative Commons license, and indicate if changes were made.

Funded by SCOAP ${ }^{3}$.

\section{References}

1. D.J. Gross, R.D. Pisarski, L.G. Yaffe, Rev. Mod. Phys. 53, 43 (1981)

2. O.K. Kalashnikov, Fort. der Phys. Prog. of Phys. 32, 525 (1984)

3. D.J. Gross, A. Neveu, Phys. Rev. D 10, 3235 (1974)

4. A. Barducci, R. Casalbuoni, M. Modugno, G. Pettini, R. Gatto, Phys. Rev. D 51, 3042 (1995)

5. C.-T. Hou, C.C.C. Mudry, Phys. Rev. Lett. 98, 186809 (2007)

6. R. Jackiw, S.-Y. Pi, Phys. Rev. Lett. 98, 266402 (2007)

7. G.W. Semenoff, Phys. Rev. Lett. 54, 2449 (1984)

8. V. Juričić, I.F. Herbut, G.W. Semenoff, Phys. Rev. B 80, 081405(R) (2009)

9. B.R. Zhou, Commun. Theor. Phys. 32, 425 (1999)

10. A. Brzoska, M. Thies, Phys. Rev. D 65, 125001 (2002)

11. J.-L. Kneur, M.B. Pinto, R.O. Ramos, E. Staudt, Phys. Rev. D 76, 045020 (2007)

12. C. Boehmer, U. Fritsch, S. Kraus, M. Thies, Phys. Rev. D 78, 065043 (2008)

13. H. Kohyama, Phys. Rev. D 77, 045016 (2008)

14. J. Feinberg, Int. J. Mod. Phys. 17, 898 (2002)

15. A.P.C. Malbouisson, J.M.C. Malbouisson, A.E. Santana, J.C. Silva, Phys. Lett. B 583, 373 (2004)

16. F.C. Khanna, A.P.C. Malbouisson, J.M.C. Malbouisson, A.E. Santana, Phys. Rev. D 85, 085015 (2012)

17. L.M. Abreu, A.P.C. Malbouisson, J.M.C. Malbouisson, Phys. Rev. D 83, 025001 (2011)

18. L.M. Abreu, A.P.C. Malbouisson, J.M.C. Malbouisson, Phys. Rev. D 84, 065036 (2011)

19. K. Gawedski, A. Kupiainen, Phys. Rev. Lett. 55, 363 (1985)
20. K. Gawedski, A. Kupiainen, Nucl. Phys. B 262, 33 (1985)

21. G. Parisi, Nucl. Phys. B 100, 368 (1975)

22. C. de Calan, P.A. Faria da Veiga, J. Magnen, R. Séneor, Phys. Rev. Lett. 66, 3233 (1991)

23. T. Inagaki, T. Kouno, T. Muta, Int. J. Mod. Phys. A 10, 2241 (1995)

24. S. Doniach, E.H. Sondheimer, Green's Functions for Solid State Physicists (W.A. Benjamin, London, 1974)

25. F.C. Khanna, A.P.C. Malbouisson, J.M.C. Malbouisson, A.E. Santana, EPL 97, 11002 (2012)

26. E.B.S. Corrêa, C.A. Linhares, A.P.C. Malbouisson, Phys. Lett. A 377, 1984 (2013)

27. C.A. Linhares, A.P.C. Malbouisson, J.M.C. Malbouisson, I. Roditi, Phys. Rev. D 86, 105022 (2012)

28. F.C. Khanna, A.P.C. Malbouisson, J.M.C. Malbouisson, A.E. Santana, Phys. Rep. 539, 135 (2014)

29. F.C. Khanna, A.P.C. Malbouisson, J.M.C. Malbouisson, A.E. Santana, Thermal Quantum Field Theory: Algebraic Aspects and Applications (World Scientific, Singapore, 2009)

30. F.C. Khanna, A.P.C. Malbouisson, J.M.C. Malbouisson, A.E. Santana, Ann. Phys. 326, 2364 (2011)

31. P. Ramond, Field Theory: A Modern Primer (Addison-Wesley, Redwood, 1990)

32. V.I. Ritus, Ann. Phys. 69, 555 (1972)

33. Soviet Phys, JETP 48, 788 (1978)

34. G. Murguía, A. Raya, Á. Sánchez, E. Reyes, Am. J. Phys. 78, 700 (2010)

35. E. Elizalde, Ann. Phys. 295, 33 (2002)

36. M. Le Bellac, Thermal Field Theory (Cambridge University Press, New York, 1996)

37. E. Elizalde, Ten Physical Applications of Spectral Zeta-Functions (Springer, Berlin, 1995)

38. L.M. Abreu, F.C. Khanna, A.P.C. Malbouisson, J.M.C. Malbouisson, A.E. Santana, Phys. Lett. A 378, 2597 (2014)

39. M.D. Scadron, F. Kleefeld, G. Rupp, EPL 80, 51001 (2007) 